\title{
Acetaldehyde-reinforcing effects: a study on oral self-administration behavior
}

\author{
Alessandra T. Peana*, Giulia Muggironi and Marco Diana \\ "G.Minardi" Laboratory of Cognitive Neuroscience, Department of Drug Sciences, University of Sassari, Sassari, Italy
}

\section{Edited by:}

Lorenzo Leggio, Brown University, USA

Reviewed by:

Carla Cannizzaro, University of

Palermo, Italy

${ }^{*}$ Correspondence:

Alessandra T. Peana, Laboratory of

Cognitive Neuroscience, Dipartimento

di Scienze del Farmaco, Università

degli Studi di Sassari, Via Muroni, 23,

07100 Sassari, Italy.

e-mail:apeana@uniss.it
Acetaldehyde (ACD) is the first metabolite of ethanol. Although, the role of ACD in ethanol addiction has been controversial, there are data showing a relationship. The objective of the current study was to further test the hypothesis that ACD itself is reinforcing. For this reason, we carried out a study on operant oral ACD self-administration. Wistar rats were trained to self-administer tap water or ACD by nose-poking in daily 30 min sessions for 15 consecutive days. Response on active nose-poke caused delivery of ACD solution or tap water, whereas responses on inactive nose-poke had no consequences. The results show that ACD maintains oral self-administration behavior and rates of active nose-pokes significantly higher than tap water. The dose-response plot for oral ACD self-administration is a "bell-shaped" curve suggesting reinforcing properties only in a limited range of doses. Furthermore, rats self-administering ACD show a deprivation effect upon $A C D$ removal and gradually reinstated active nose-poke response when ACD was reintroduced. Overall, this study shows that ACD is orally self-administered and further supports the hypothesis that $A C D$ possesses reinforcing properties, which suggests that some of the pharmacological effects attributed to ethanol may result from its biotransformation into $A C D$, thereby supporting an active involvement of $A C D$ in ethanol addiction.

Keywords: acetaldehyde, reinforcing effects, oral self-administration behavior, Wistar rats

\section{INTRODUCTION}

Although well known for its toxicity (Novartis Foundation, 2007), acetaldehyde (ACD) is recognized by the Food and Drug Administration as a safe compound (FDA, 2008). ACD is the first metabolite of ethanol and is also a major component of tobacco smoke (Talhout et al., 2007). In addition, ACD is also present in alcoholic drinks such as beer, wine, sake, and whiskey and in very small amounts in some fermented foods (Liu and Pilone, 2000; Novartis Foundation, 2007; Talhout et al., 2007). ACD, however, first evokes to pharmacologists and physicians its indirect use as deterrent to discourage drinking in alcoholic patients. In fact, disulfiram and other aldehyde dehydrogenases inhibitors induce elevated blood ACD concentrations and elicit the strong aversive syndrome, known as flushing reaction (Quertemont et al., 2005; Suh et al., 2006). On the other hand, some authors reported that patients benefited by taking low doses of ethanol when under disulfiram treatment (Chevens, 1953; Brown et al., 1983). However, the interest in $\mathrm{ACD}$ as a compound with potential addictive effects has been revitalized in the last decade by a number of studies, suggesting the possibility that ethanol effects could be mediated by its first metabolite, ACD (Quertemont et al., 2005). Experimental evidence supports the notion that motivational properties of ethanol are mediated by ACD (McBride et al., 2002; Font et al., 2006; Peana et al., 2008a, 2009). Moreover, a wealth of studies suggest that ACD itself could produce positive reinforcing effects (Brown et al., 1979; Myers et al., 1984; Smith et al., 1984; Takayama and Uyeno, 1985; Quertemont and De Witte, 2001; Rodd-Henricks et al., 2002). Interestingly, ACD itself and as a consequence of the metabolism of ethanol, activates neuronal firing of dopaminergic neurons in the VTA (Foddai et al., 2004) and stimulates dopamine transmission in the nucleus accumbens (Melis et al., 2007; Enrico et al., 2009). Further, Vinci et al. (2010) reported that ACD elicits extracellular signal-regulated kinase (ERK) phosphorylation in the rat Nacc and extended amygdala (Vinci et al., 2010). Overall, these evidences contribute to the suggestion that ACD is a compound with intrinsic motivational and rewarding effects.

The objective here is to further test the hypothesis that ACD itself could be reinforcing. For this reason, we carried out a study on acquisition, maintenance (stable baseline), and deprivation effect in ACD-drinking behavior.

\section{MATERIALS AND METHODS}

The study was carried out in accordance with current Italian legislation [D.L. 116, 1992] which allows experimentation on laboratory animals only after submission and approval of a research project to the Ministry of Health (Rome-Italy), and in strict accordance with European Council directives on the matter [n. 2007/526/CE]. All possible efforts were made to minimize animal pain and discomfort and to reduce the number of experimental subjects.

\section{ANIMALS}

Male Wistar rats (Harlan, Udine, Italy), weighing 175-225 g, were housed in groups of two and maintained under controlled environmental conditions (temperature $22 \pm 2^{\circ} \mathrm{C}$; humidity $60-65 \%$ ) on a reverse 12-h light/dark cycle (light on at 18:00 hours; off at 06:00 hours). In order to limit animal stress, the rats were housed in the same room where the operant chambers were located. Standard laboratory rat chow and tap water (two bottles) were available ad libitum in the home cage, with the exceptions noted below. All training and experimental sessions were conducted during the 
dark phase of the cycle (Testa and Badiani, 2008). We performed the experiment during the dark phase, when is more evident the drinking behavior (Kamdar et al., 2007).

\section{DRUGS}

Acetaldehyde (Sigma-Aldrich, Milano, Italy) was dissolved in tap water as w/v. All dilutions were freshly prepared before every experimental session. So as to avoid ACD evaporation, we prepared the solutions keeping the preparation beaker under ice. In addition, ACD was always used in this diluted form which reduced surface tension.

\section{APPARATUS}

Training and testing were conducted in modular operant chambers located in ventilated sound-attenuating environmental cubicles (Med Associates Inc, USA). Each chamber was equipped with a non-retractable drinking reservoir (capacity $0.50 \mathrm{ml}$ ) and two nosepoke holes, located at $3 \mathrm{~cm}$ to the left and right of the reservoir. As discriminative stimuli, a white light was placed over the active hole and a red light over the inactive one. Only the active nose-poke set off the dipper delivering the solution $(0.1 \mathrm{ml})$ and was recorded. Explorations on the other inactive nose-poke were also recorded. This served to control for specificity of response in the operant chamber. The availability of liquid was signaled by a white house light placed on the wall in front of the liquid delivery system and would light up for the duration of liquid delivery. Following each delivery, there was a 2 -s time-out period during which response had no consequences and the white light placed over the active hole would go off. An infrared head detector was located in the reservoir and recorded all signals during the whole session lasting $30 \mathrm{~min}$. Chambers were interfaced to a computer equipped with software that programmed sessions as well as data recording.

\section{OPERANT PROCEDURES}

Rats $(n=11)$ learned how to obtain ACD solution by nose-poke. During the self-administration period, we repeatedly evaluated active and inactive nose-pokes as well as ACD intake in relation to each rat's weight $(\mathrm{g} / \mathrm{kg})$ for each daily 30 -min session. For operant ACD self-administration (full range $0.1-0.2 \%$ ), rats were trained to nose-poke on a fixed-ratio 1 schedule of reinforcement, where each response resulted in $0.1 \mathrm{ml}$ solution delivery. One day before (pre-training) and for the first 2 days of training, tap water availability in the home cage was restricted to $5 \mathrm{~min} /$ day in order to facilitate acquisition of operant response. From this point onwards, tap water was continuously available in the home cage. During this time ( 3 days, deprived +3 days, not deprived), rats were permitted to nose-poke explore for $0.1 \% \mathrm{ACD}$ solution Starting on day $7, \mathrm{ACD}$ solution concentration was gradually increased in stages from 0.12 to $0.14,0.16$, and 0.18 ending up at the final concentration of $0.2 \%$. Following completion (11 days) of the ACD training the rats were trained to nose-poke explore for another 4 days: this served to reach a stable responding baseline (maintenance). Inactive nose-poke responses were recorded during all testing phases as a measure of non-specific behavioral response. An additional group of five rats was included and these were given the opportunity to orally self-administer tap water and trained under the same experimental conditions as the ACD group.
For the study of ACD dose-response another group of rats $(n=5)$ was trained in a daily 30 min session to ACD self-administer at concentrations starting from day 7 until 11 at $0.1 \%$ and then gradually increased through $0.2-3.2 \%$, every 5 days for each concentration session.

In the extinction paradigm, subjects $(n=5)$ are trained to orally self-administer ACD until stable self-administration patterns are achieved, at which point ACD is removed. Extinction testing sessions are identical to training sessions except that there is no $\mathrm{ACD}(0.2 \%)$. Our extinction period lasted five consecutive days and during this time, responses at the active nose-poke set off the delivery mechanism. Following the last extinction session, began the deprivation-effect sessions. These sessions lasted $30 \mathrm{~min}$ under conditions identical to those during the self-administration session with availability of ACD solution at $0.2 \%$ and proceeded for a further 5 days. Response at the inactive nose-poke was constantly recorded to monitor possible non-specific behavioral effects.

\section{MEASUREMENTS OF BLOOD AND BRAIN ACETALDEHYDE LEVELS}

Immediately after the final session of self-administration, animals were anesthetized with ketamine and medetomidine $(75+0.5 \mathrm{mg} /$ $\mathrm{kg} / \mathrm{ml}$, i.p.) and about $10 \mathrm{~min}$ afterward blood samples and brain were taken. For analysis, a 1-ml aliquot of rat blood was diluted with $1 \mathrm{ml}$ of cold milliQ water in $10 \mathrm{ml} \mathrm{HS}$-vials (Hewlett-Packard). The brains, on the other hand, were homogenized with $1 \mathrm{ml}$ of cold milliQ water in $10 \mathrm{ml}$ HS-vials (Hewlett-Packard) for analysis. The vials were placed in a heating block at $45^{\circ} \mathrm{C}$ for $10 \mathrm{~min}$. The samples were analyzed on HS-GC-FID system with a Dani 86.50 HSSautosampler, and a Hewlett-Packard gas chromatography HP 6890 Plus. An Econo CAP EC-5 (Alltech, Italy) capillary column used was ( $30 \mathrm{~m}, 0.53 \mathrm{~mm}$ i.d., $1.2 \mu \mathrm{m}$ d.f.). The injection port temperature was maintained at $250^{\circ} \mathrm{C}$. GC oven temperature was maintained at $45^{\circ} \mathrm{C}$ in isothermal conditions for $8 \mathrm{~min}$. The flow rate of helium carrier gas was $6.1 \mathrm{ml} / \mathrm{min}$ and the FID temperature was maintained at $250^{\circ} \mathrm{C}$. HS parameters were: $75^{\circ} \mathrm{C}$ manifold temperature, $150^{\circ} \mathrm{C}$ transferline temperature, 1.57 PSI carrier gas pressure, 1 min vial pressurization time, and $1 \mathrm{ml}$ injection volume of headspace gas.

\section{STATISTICAL ANALYSIS}

Data were analyzed by one-way analysis of variance (ANOVA) or two-way ANOVA for repeated measures and are expressed as mean \pm S.E.M. Post hoc comparisons, using Tukey's Honestly Significant Difference (HSD) test, were undertaken if a significant effect was found $(p<0.05)$. In addition, for each individual grouping, nose-poke discrimination was determined by type (active or inactive) $\times$ session multivariate ANOVA (MANOVA) with $F$-values derived from Wilk's Lambda.

\section{RESULTS}

Figure 1, illustrates that rats $(n=11)$ showed a higher number of responses for $\mathrm{ACD}$ solution versus tap water $(n=5)$ during the acquisition session [active nose-poke: $F(1.14)=4.69, p=0.048$ ]; session: $F(14.196)=8.92, p<0.00001$ but not an active nosepoke $\times$ session interaction effect]. In addition, nose-poke discrimination (active against inactive nose-pokes) was present for the major part of acquisition sessions [Wilks' Lambda: 0.000793; Rao's R: 420.1407; $p<0.0001$; one-way MANOVA analysis]. 
Further, Figure 2, shows the corresponding ACD intake (mg/ $\mathrm{kg}$ ) as a function of active nose-poke in the ACD same grouping. One-way ANOVA on the average intake during the 15 sessions revealed a significant intake effect $[F(14.150)=9.01, p<0.00001]$. In general, the animals consumed more ACD with respect to the first (tap water deprived) and fourth (not deprived) sessions of self-administration. Consumatory responses, was measured, also by head entries throughout the session period (about 170\% with respect to total infusions).

For the analysis of ACD dose-response $(n=5)$, the number of active nose-pokes during the final 3 ACD self-administration sessions was averaged to obtain a mean value for ACD self-administration (Figure 3). One-way ANOVA on the average number of active nose-pokes explorations revealed a significant effect of concentration $[F(6.14)=6.54, p=0.0018]$. Post hoc comparisons indicated that the $0.2,0.4,0.8$, and $1.6 \%$ ACD concentrations induced significantly more exploration of active nose-pokes $(p<0.05)$ with respect to the 0.1 and $3.2 \%$ concentrations, as well as with respect

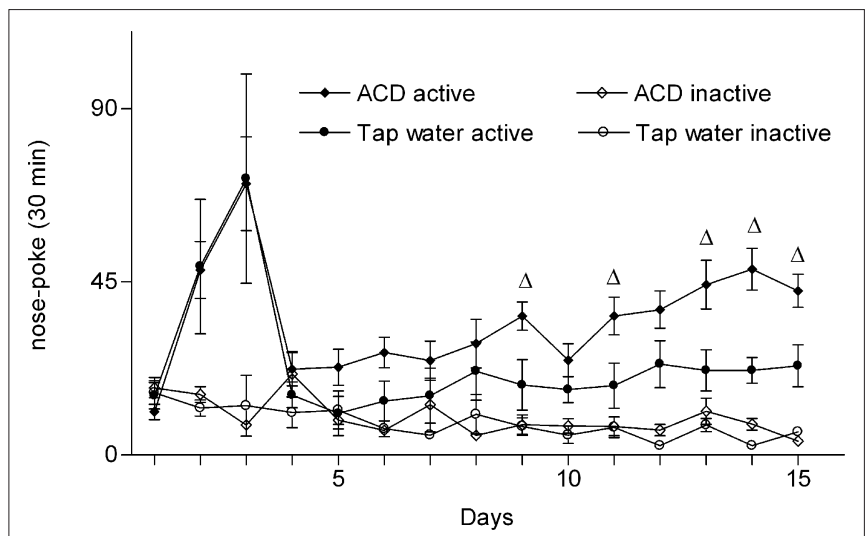

FIGURE 1 | Oral ACD self-administration behavior. Responses per session (30 $\mathrm{min}$ ) on the active and inactive nose-pokes by rats trained for acquisition of oral tap water or ACD self-administration. ${ }^{\Delta} p<0.05$ between tap water and $A C D$, active nose-pokes (two-way ANOVA for repeated measures and Tukey's post hoc test). Data are mean \pm SEM.

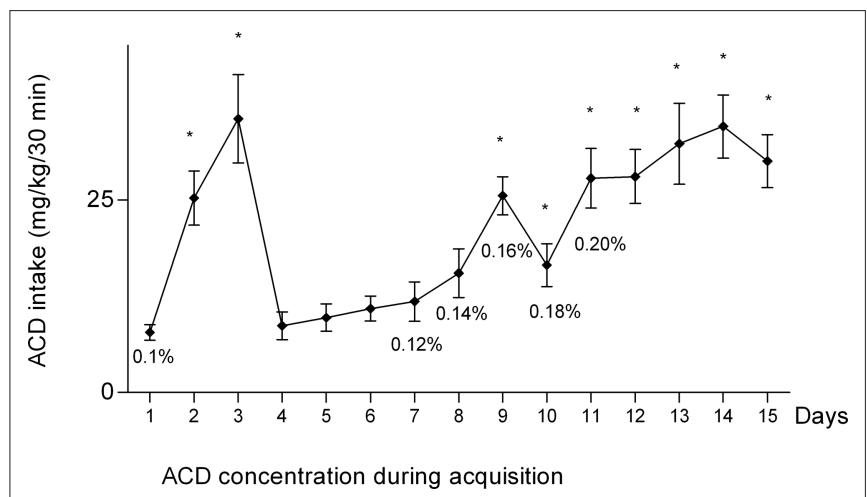

FIGURE 2 |Acetaldehyde intake expressed in $\mathrm{mg} / \mathrm{kg}$ during the above (Figure 1) operant session (30 $\mathrm{min}$ ) as function of active nose-poke in the same ACD group. ${ }^{*} p<0.05$ with respect to the $1^{\circ}$ and $4^{\circ}$ day of $A C D$ session (one-way ANOVA and Tukey's post hoc test). Data are mean \pm SEM. to tap water $(n=5)$. Moreover, responses on the active nose-poke showed no variation between ACD solution at 0.1 and $3.2 \%$, and tap water (Figure 3).

Following completion of ACD training (maintenance or stable baseline of drinking), the rats $(n=5)$ were subjected to a period of ACD extinction for 5 days and afterward resumed ACD self-administration for 5 days. The amount of response on the now reactivated nose-poke was used to reflect the motivation for ACD-drinking behavior. During these sessions the rats responded significantly more on the active than the inactive nose-poke. Our MANOVA data show that the subjects discriminated between active and inactive nose-pokes before, [Wilks' Lambda: 0.031; Rao's R: 25.0065; $p=0.004$ ], and after extinction session [Wilks' Lambda: 0.023; Rao's R: $33.732 ; p=0.002$ ], but not during the 5-day extinction session (Figure 4).

As can be seen in Table 1, following the final session of oral ACD self-administration, ACD levels in blood were increased up to $62 \%(n=8)$ with respect to the tap water group $(n=4)$. One way ANOVA showed a significant effect of treatment $[F(1.20)=2.30$, $p<0.05]$. In contrast, brain ACD levels were identical in the two grouping (data not shown).

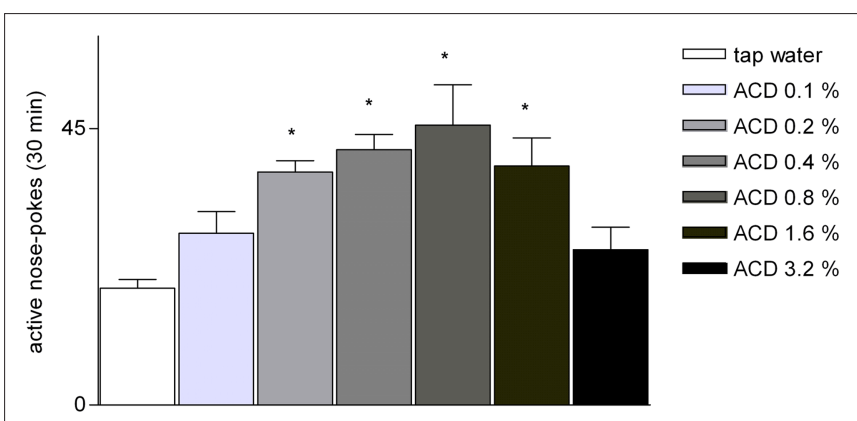

FIGURE 3 | Average number of active nose-pokes during the last three sessions of oral self-administration as a function of ACD concentration (each ACD concentration was tested for $\mathbf{5}$ days) in Wistar rats. Asterisk indicate significant difference $(p<0.05)$ with Tukey's test. Data are mean \pm SEM .

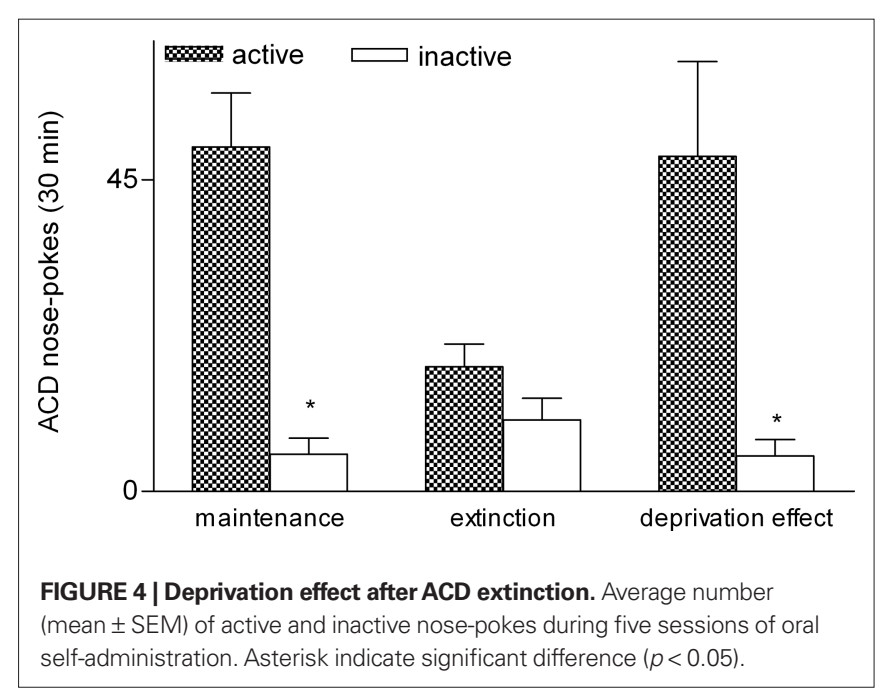


Table 1 | Acetaldehyde blood levels.

\begin{tabular}{lcll}
\hline Treatment & $\begin{array}{l}\text { ACD intake } \\
(\mathbf{m g} / \mathbf{k g})\end{array}$ & $\begin{array}{l}\text { ACD level } \\
(\mathbf{m g} / \mathbf{m l})\end{array}$ & \% Increase \\
\hline Saline/ACD & $20.00 \pm 2.11$ & $0.0073 \pm 0.0010$ & 62 \\
Saline/tap water & & $0.0045 \pm 0.0016$ & \\
\hline
\end{tabular}

Results are presented as the mean \pm SEM.

\section{DISCUSSION}

Acetaldehyde addicting effects are still under debate. Therefore, the aim of the current study was to further test the hypothesis that ACD itself is reinforcing. The results of these experiments show (1) that ACD elicits a greater number of active nose-pokes for ACD versus tap water during the acquisition of oral self-administration behavior and (2) discriminatory behavior between active and inactive nose-pokes. However, oral ACD self-administration did not modify the number of the inactive nose-pokes with respect to the tap water grouping; indicating an absence of non-specific behavioral effects as well as an absence of motor properties. Without any prior operant experience, the rats learned to discriminate the active from the inactive nose-poke by day 5 of the initial ACD acquisition period. As well, from day 9 of acquisition, the active nose-pokes for oral ACD self-administration were significantly higher than for tap water. Oral ACD self-administration $(0.2 \%)$ induced an average intake of about $20 \mathrm{mg} / \mathrm{kg} / \mathrm{session}$ similar to the intragastric dose of ACD $(20 \mathrm{mg} / \mathrm{kg})$ previously found to induce conditioned placepreference in Wistar rats (Peana et al., 2008a, 2009).

In addition, ACD self-administration shows a "bell-shaped" curve, suggesting the presence of reinforcing properties within a limited dosage range. Rats orally self-administered ACD at concentrations as low as 0.2 up to $1.6 \%$ with the lowest $(0.1 \%)$ and highest concentrations (3.2\%) not self-administered. The decrease in oral self-administrations as the concentrations increased to over $0.8 \%$ might be due to the higher doses producing a greater effect per delivery so that fewer nose-pokes were needed to give the same effect. In addition, the lack of oral ACD self-administration at the higher concentrations could be due to development of acute tolerance to ACD or a result of the toxic/aversive effects of the substance (Quertemont and De Witte, 2001). The "bell-shaped" plot for ACD was somewhat similar to the dose-response plot seen for ACD and ethanol in VTA self-administration studies (Rodd-Henricks et al., 2000, 2002). The dose-response data indicates that the lowest limit for producing a reinforcing effect in ACD is around $0.2 \%$. Furthermore, rats that self-administered $0.2 \%$ of ACD, showed extinction behavior with a loss of nose-poke discrimination and subsequently, after ACD restoration, a gradual reinstatement of active nose-poke response.

The innovation of the present report is that ACD was orally self-administered. In particular, during the final session of ACD self-administration, consumatory responses were confirmed by a relevant increase (62\%) in ACD blood levels with respect to the tap water animal grouping. Our findings on blood ACD levels are of importance because the physiochemical properties of ACD have been the focus of continuing interest and controversy regarding the its neurobehavioral actions.
On the other hand, we found no differences in ACD levels in the brain between the two grouping, probable due to low brain ACD penetration. In this context, ACD was actually consumed at very low doses considering that the amount in $\mathrm{mg} / \mathrm{kg}$ was ingested over a 30-min session and not in a single bolus. We cannot exclude that other experimental protocols might give different results; indeed, several reports have pointed out the difficulties affecting ACD measurement in the brain, particularly due to the low levels usually encountered and very few studies have reported detectable brain ACD levels after peripheral ACD administration. Overall, ACD determination is difficult and compromised by its rapid conversion to acetate, via aldehyde dehydrogenase (Deng and Deitrich, 2008). In fact, rapid ACD oxidation, may markedly affect the recovery of low ACD levels. Moreover, ACD, like other volatiles, is eliminated by breath crossing the alveolar-capillary membrane of the lungs (Novartis Foundation, 2007). Nevertheless, ACD easily reacts with many other molecules by adduction, condensation and polymerization, which are important in the brain, and these could be plausible explanations for why we did not detect ACD in the brain. Some authors reported that cerebral venous blood ACD (not brain) levels were detected after a high ACD dose (Quintanilla and Tampier, 2003). Moreover, studies, which have utilized high doses of ACD, demonstrated that ACD, analyzed by HPLC, was present in rat brain after a single intraperitoneal injection of ACD (Heap et al., 1995). It is important to note that we analyzed brain samples by gas chromatography with the headspace.

The exact mechanism underlying ACD behavioral effects remains largely unknown. ACD could play a mediatory role on the neurobehavioral consequences observed after ethanol administration. Even the mechanism of ethanol actions is still debated although multiple molecular targets have been identified. Nevertheless, there have been significant advancements in understanding of ACD central effects in recent years. Previous findings of our research group showed that the peripheral competitive inhibitor of alcohol dehydrogenase, 4-methylpyrazole, decreased ethanol-induced conditioned place preference and oral ethanol self-administration in Wistar rats (Peana et al., 2008a,b). These findings were supported by the efficacy of selective ACD sequestrating agents, D-penicilamine and L-cysteine, to prevent ethanol- and ACD-induced conditioned place preference in Wistar rats (Peana et al., 2008a, 2009). In agreement, L-cysteine inhibited oral ethanol self-administration in Wistar rats (Peana et al., 2010) and D-penicilamine reduced voluntary ethanol consumption (Font et al., 2006). The present observations that ethanol-derived ACD and ACD elicits positive motivational properties are in agreement with recent studies reporting that intragastric ACD and ethanolderived ACD could share with addictive drugs the property to induce ERK phosphorylation in the Sprague-Dawley's rat Nacc and extended amygdala (Vinci et al., 2010). Moreover, another study demonstrates that blockade of MEKs prevents ACD elicited conditioned place preference in Sprague-Dawley rats (Spina et al., 2010). Furthermore, previous work reported that ACD and ethanol-derived ACD increased the firing rate of dopaminergic neurons in VTA in Wistar rats (Foddai et al., 2004; Enrico et al., 2009). In patch-clamp studies, Melis et al. (2007) showed that ACD increased the inward current in DA neurons, suggesting a direct effect for ACD on dopaminergic neural membranes. 
Acetaldehyde could exert its effects by reacting with endogenous neurotransmitters and proteins to form tetrahydro- $\beta$-carboline and tetrahydroisoquinolines alkaloids, involved in ethanol consumption (Davis and Walsh, 1970; Merchior, 1979; Myers et al., 1985; Matsuzawa et al., 2000; Quertemont and Tambour, 2004; Talhout et al., 2007; Rodd et al., 2008), eventually leading to development of addiction.

In summary, the results of this study indicate that ACD shares with ethanol the ability to induce an operant oral self-administration behavior. These data further support the

\section{REFERENCES}

Brown, Z. W., Amit, Z., and Rockman, G. E. (1979). Intraventricular selfadministration of acetaldehyde, but not ethanol, in naïve laboratory rats. Psychopharmacology 64, 271-276.

Brown, Z. W., Amit, Z., Smith, B. R., Sutherland, E. A., and Selvaggi, N. (1983). Alcohol-induced euphoria enhanced by disulfiram and calcium carbimide. Alcohol. Clin. Exp. Res. 7, 276-278.

Chevens, L. C. F. (1953). Antabuse addiction. Br. J. Med. 1, 1450-1451.

Davis, V. E., and Walsh, M. J. (1970). Alcohol addiction and tetrahydropapaveroline. Science 169, 1105-1106.

Deng, X., and Deitrich, R. A. (2008). Putative role of brain acetaldehyde in ethanol addiction. Curr. Drug Abuse Rev. 1, 3-8.

Enrico, P., Sirca, D., Mereu, M., Peana, A. T., Lintas, A., Golosio, A., and Diana, M. (2009). Acetaldehyde sequestering prevents ethanol-induced stimulation of mesolimbic dopamine transmission. Drug Alcohol Depend. 100, 265-271.

FDA-U.S. Food and Drug Administration. (2008). Code of Federal Regulations. Washington, DC: FDA, 21CFR172.515.

Foddai, M., Dosia, G., Spiga, S., and Diana, M. (2004). Acetaldehyde increases dopaminergic neuronal activity in the VTA. Neuropsychopharmacology 29, 530-536.

Font, L., Aragon, C. M., and Miquel, M. (2006). Voluntary ethanol consumption decreases after the inactivation of central acetaldehyde by d-penicillamine. Behav. Brain Res. 171, 78-86.

Heap, L., Ward, R. J., Abiaka, C., Dexter, D., Lawlor, M., Pratt, O., Thomson, A., Shaw, K., and Peters, T. J. (1995). The influence of brain acetaldehyde on oxidative status, dopamine metabolism and visual discrimination task. Biochem Pharmacol. 50, 263-270.

Kamdar, N. K., Miller, S. A., Syed, Y. M., Bhayana, R., Gupta, T., and Rhodes,
J. S. (2007). Acute effects of naltrexone and GBR 12909 on ethanol drinking-in-the-dark in C57BL/6J mice. Psychopharmacology 192, 207-217.

Heap, L., Ward, R. J., Abiaka, C., Dexter, D., Lawlor, M., Pratt, O., Thomson, A., Shaw, K., and Peters, T. J. (1995). The influence of brain acetaldehyde on oxidative status, dopamine metabolism and visual discrimination task. Biochem. Pharmacol. 50, 263-270.

Liu, S., and Pilone, G. J. (2000). An overview of formation and roles of acetaldehyde in winemaking with emphasis on microbiological implications. Int. J. Food Sci. Technol. 35, 49-61.

Matsuzawa, S., Suzuki, T., and Misawa, M. (2000). Involvement of mu-opioid receptor in the salsolinol-associated place preference in rats exposed to conditioned fear stress. Alcoholism: Clinical and Experimental Research 24, 366-372.

McBride, W. J., Li, T. K., Deitrich, R. A., Zimatkin, S., Smith, B. R., and RoddHenricks, Z. A. (2002). Involvement of acetaldehyde in alcohol addiction. Alcohol. Clin. Exp. Res. 26, 114-119.

Melis, M., Enrico, P., Peana, A. T., and Diana, M. (2007). Acetaldehyde mediates alcohol activation of the mesolimbic dopamine system. Eur. J. Neurosci. 26, 2824-2833.

Merchior, C. L. (1979). Interaction of salsolinol and tetrahydropapaveroline with catecholamines. Alcohol. Clin. Exp. Res. 3, 364-367.

Myers, W. D., Ng, K. T., and Singer, G. (1984). Effects of naloxone and buprenorphine on intravenous acetaldehyde self-injection in rats. Physiol. Behav. 33, 449-455.

Myers, W.D., Ng, K. T., Singer, G., Smythe, G. A., and Duncan, M. W. (1985). Dopamine and salsolinol levels in rat hypothalami and striatum after schedule-induced self-injection (SISI) of ethanol and acetaldehyde. Brain Res. 358, 122-128.

Novartis Foundation. (2007). Acetaldehyde-Related Pathology:

hypothesis that ACD by itself holds reinforcing effects possibly via potentiation of the activity dopaminergic neurons in the mesolimbic system.

\section{ACKNOWLEDGMENTS}

This work was supported by grants from the "Fondazione Banco di Sardegna" (596/2008.0078) to Alessandra T. Peana and PRIN (MIUR, 2006057754) to Marco Diana. The authors wish to thank the Eng. Sergio Sanna for the help in the software program utilized for the experiments.

Bridging the Trans-disciplinary Divide, Symposium 285. Chichester: John Wiley \& Sons.

Peana, A. T., Enrico, P., Assaretti, A. R., Pulighe, E., Muggironi, G., Nieddu, M., Piga, A., Lintas, A., and Diana, M. (2008a). Key role of ethanol-derived acetaldehyde in the motivational properties induced by intragastric ethanol: a conditioned place preference study in the rat. Alcohol. Clin. Exp. Res. 32, 249-258.

Peana, A. T., Assaretti, A. R., Muggironi, G., Enrico, P., and Diana, M. (2008b). The Peripheral Competitive Inhibitor of Alcohol Dehydrogenase, 4-Methylpyrazole, Reduces OralEthano Self-Administration. ESBRA2008 Nordmann Award Meeting Animal Models in Alcohol Research. September 26-27, 2008, Cagliari, Sardinia, Italy.

Peana, A. T., Assaretti, A. R., Muggironi, G., Enrico, P., and Diana, M. (2009) Reduction of ethanol-derived acetaldehyde induced motivational properties by L-cysteine. Alcohol. Clin. Exp. Res. 33, 43-48.

Peana, A. T., Muggironi, G., Calvisi, G., Enrico, P., Mereu, M., Nieddu, M., Boatto, G., and Diana, M. (2010) L-cysteine reduces oral ethanol selfadministration and reinstatement of ethanol-drinking behavior in rats. Pharmacol. Biochem. Behav. 94, 431-437.

Quertemont, E., and De Witte, P. (2001). Conditioned stimulus preference after acetaldehyde but not ethanol injections. Pharmacol. Biochem. Behav. 68 449-454.

Quertemont, E., and Tambour, S. (2004). Is ethanol a pro-drug? The role of acetaldehyde in the central effects of ethanol. Trends Pharmacol. Sci. 25, 130-134.

Quertemont, E., Tambour, S., and Tirelli, E. (2005). The role of acetaldehyde in the neurobehavioral effects of ethanol: a comprensive review of animal studies. Prog. Neurobiol. 75, 247-274.
Quintanilla, M. E., and Tampier, L. (2003). Acetaldehyde-reinforcing effects: differences in low-alcoholdrinking (UChA) and high-alcoholdrinking (UChB) rats. Alcohol 31, 63-69.

Rodd, Z. A., Oster, S. M., Ding, Z. M., Toalston, J. E., Deehan, G., Bell, R. L. Li, T. K., and McBride, W. J. (2008) The reinforcing properties of salsolinol in the ventral tegmental area: evidence for regional heterogeneity and the involvement of serotonin and dopamine. Alcohol. Clin. Exp. Res. 32, 230-239.

Rodd-Henricks, Z. A., McKinzie, D. L., Murphy, J. M., and McBride, W. J. (2000). Regional heterogeneity for the intracranial self-administration of ethanol within the ventral tegmental area of female Wistar rats. Psychopharmacology 149, 217-224

Rodd-Henricks, Z. A., Melendez, R. I., Zaffaroni, A., Goldstein, A., McBride, W. J., and Lu, T. K. (2002). The reinforcing effects of acetaldehyde in the posterior ventral tegmental area of alcohol-preferring rats. Pharmacol. Biochem. Behav. 72, 55-64.

Smith, B. R., Amit, Z., and Splawinsky, J. (1984). Conditioned place preference induced by intraventricular infusions of acetaldehyde. Alcohol 1 , 193-195.

Spina, L., Longoni, R., Vinci, S., Ibba, F., Peana, A. T., Muggironi, G., Spiga, S., and Acquas, E. (2010). Role of dopamine D1 receptors and extracellular signal regulated kinase in the motivational properties of acetaldehyde as assessed by place preference conditioning. Alcohol. Clin. Exp. Res. 34, 1-10.

Suh, J. J., Pettinati, H. M., Kampman, K. M., and O'Brien, C. P. (2006). The status of disulfiram: a half of a century later. J. Clin. Psychopharmacol. 26, 290-302.

Takayama, S., and Uyeno, E. T. (1985). Intravenous self-administration of ethanol and acetaldehyde by rats. Yakubutsu Seishin Kodo 5, 329-334. 
Talhout, R., Opperhuizen, A., and van Amsterdam, J. G. C. (2007). Role of acetaldehyde in tobacco smoke addiction.Eur. J. Neuropsychopharmacol. 17, 627-636.

Testa, A., and Badiani, A. (2008). Environmental modulation of alcohol self-administration in the rat. Neuroscience. Program/Poster: 564.26/ II2. http://www.abstractsonline.com/ PLAN/SSResults.aspx
Vinci, S., Ibba, F., Longoni, R., Spina, L., Spiga, S., and Acquas, E. (2010). Acetaldehyde elicits ERK phosphorylation in the rat nucleus accumbens and extended amygdale. Synapse. Apr 29. [Epub ahead of print].

Conflict of Interest Statement: The authors declare that the research was conducted in the absence of commercial or financial relationships that could be construed as a potential conflict of interest.

Received: 17 June 2010; paper pending published: 30 June 2010; accepted: 06 July 2010; published online: 16 September 2010. Citation: Peana AT, Muggironi $G$ and Diana M (2010) Acetaldehyde-reinforcing effects: a study on oral self-administration behavior. Front. Psychiatry 1:23. doi: 10.3389/fpsyt.2010.00023
This article was submitted to Frontiers in Addictive Disorders, a specialty of Frontiers in Psychiatry.

Copyright (C) 2010 Peana, Muggironi, and Diana. This is an open-access article subject to an exclusive license agreement between the authors and the Frontiers Research Foundation, which permits unrestricted use, distribution, and reproduction in any medium, provided the original authors and source are credited. 